\title{
Based On Intrinsic Mode Function Energy Tracking Method of Circuit Breaker Vibration Signal Feature Extraction Studies
}

\author{
Yi-Hang Sun ${ }^{1}$ \\ ${ }^{1}$ Beijing Institute of Mechanical Equipment, Beijing, China 100854
}

\begin{abstract}
In order to detect a mechanical type of structural failure of the circuit breaker, the characteristics of the circuit breaker mechanical vibration signal is analyzed in this paper. A combination of medium voltage circuit breaker based on empirical mode decomposition (EMD) amount of energy and support vector machine (SVM) theory vibration signal feature vector extraction and analysis of fault classification method is proposed. First, the vibration signal of the circuit breaker is decomposed by EMD, then intrinsic mode function (IMF) is obtain. The major fault feature information intrinsic mode functions the amount of energy of the component is obtained by discrete sampling points and the amount of energy. Using the amount of energy of IMF component as a feature vector, the failure of the test sample signal as input feature vector into trained "BT-SVM" support vector machine classification mechanism for fault classification. The differences and fault type of vibration signals can be identified by this method through the experimental analysis.
\end{abstract}

\section{Introduction}

A circuit breaker is very critical distribution systems important equipment. With the distribution network automation and reliability requirements increase, the circuit breaker itself is also increasing reliability requirements [1-3].

In the operation process of the circuit breaker contains a lot of important status information when a fault message appears fault signal when the feature vector extraction is critical fault signal analysis in the current analysis method is only suitable for the fast Fourier transform analysis of stationary signals, while the mechanical vibration signal is non-stationary nature, must be found suitable for the analysis of non-stationary signal processing method. Wavelet analysis, short-time Fourier transform methods have their limitations, it can not be good for non-stationary signal analysis, so this article will EMD method is applied to the circuit breaker vibration signal feature vector extraction field, it is a new suitable for analyzing non-stationary signal analysis methods. EMD is a Chinese-American NordenE .Huang et al proposed [4-7], it is suitable to handle nonlinear and non-stationary signals, it has a high signal to noise ratio, can decompose complex signals, resulting in finite IMF component, IMF component contains the original signal at different time scales local characteristic signal. By analyzing the local signal characteristics including IMF to accurately extract the important features of the original signal, each IMF frequency components contained in the signal sampling frequency is not correlated with the signal itself, it is adaptive [8-9].

\section{Vibration Signal EMD}

If there are any complex signals are composed of a plurality of different IMF, each IMF can be linear or non-stationary, non-linear, each intrinsic mode function must meet the following two conditions: First, the entire data segment, the number of extreme points and the number of zero crossings must be equal or differ at most no more than one; secondly, at any time, by the formation of the local maxima and the envelope formed by the local minimum point the average value of the lower envelope is zero, i.e., the lower envelope locally symmetric with respect to the time axis.

(1) determine the signal all the local extreme points, and then use the left and right cubic spline local maxima joined together to form the upper envelope.

(2) a cubic spline in all the local minimum points are connected to form the lower envelope, the upper and lower envelope should envelop all the data points.

(3) the average envelope denoted $m_{1}(t)$, is given by:

$$
x(t)-m_{1}(t)=h_{1}(t)
$$

(4) If ${ }^{h_{1}(t)}$ is not the IMF conditions, as the original data to, repeating steps (1) to (3), to obtain the envelope of the average $m_{11}(t)$ of the judgment meets the IMF conditions: $h_{11}(t)=h_{1}(t)-m_{11}(t)$, if not met, then the loop 
times $k$, to obtain $h_{1(k-1)}(t)-m_{1 k}(t)=h_{1 k}(t)$, so as to satisfy IMF conditions: ${ }^{h_{k}}(t)$.Mind ${ } c_{1}(t)=h_{1 k}(t), c_{1}(t)$ for the first signal component to meet IMF conditions of $^{x(t)}$.(5) $c_{1}(t)$ separated from ${ }^{x(t)}$ the obtained:

$$
r_{1}(t)=x(t)-c_{1}(t)
$$

As ${ }^{r_{1}(t)}$ raw data repeating steps (1) to (4), to obtain a second component $c_{2}$ of $x(t)$ which satisfy the conditions of IMF. Repeat the cycle times $n$ to obtain the signal component of $x(t)$ which satisfy the conditions of IMF. "Screening" process by limiting the stopping criterion $S_{d}$ processing result of two successive magnitude of the difference between the standard to achieve the decomposition is stopped when less than 0.3 .

$$
S_{d}=\sum_{t=0}^{T} \frac{\left|\left(h_{(k-1)}(t)-h_{k}(t)\right)\right|^{2}}{h_{k}^{2}(t)}
$$

\section{3. "Binary tree" Support Vector Machine}

In this paper, two-dimensional space can be divided into two kinds of support vector machines briefing formation process. Figure (1) shows, rectangular and elliptical dot points represent two types of data samples, $\mathrm{H}$ is classified lines, $\mathrm{H} 0$ and $\mathrm{H} 1$ intermediate samples were classified by a sample line and parallel to the nearest classification line, the spacing between them Be the classification interval. For linearly separable problems, there always exists a hyper plane $\omega \cdot x+b=0$ two types of training samples can be completely separated, H0 and H1 is the sample points on the support vector [21].

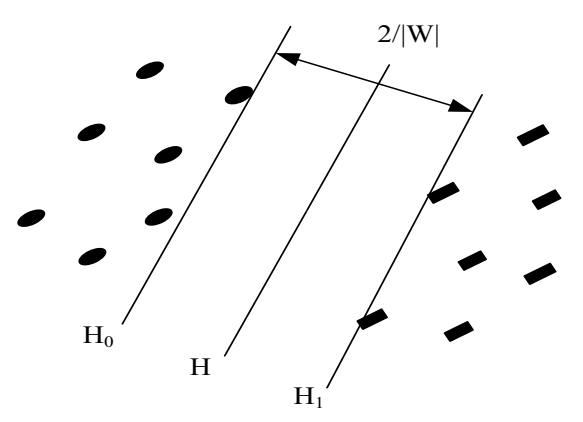

Fig1 Classification line based on 2-D linear separability

But the reality is that the majority of non-linear, and for the case of non-linear, SVM generally taken to introduce slack variables $\xi_{i}$ and punishment factor $\mathrm{C}$ form hyperplane constraints:

$$
y_{i}[(\omega \cdot x+b)] \geq 1-\xi_{i}
$$

Where $\omega$ is the surface normal vector classification for classification threshold. In the generalized optimal hyper plane problem by introducing Lagrange multipliers, build generalized optimal hyper plane problem can be converted to solve the dual quadratic programming problems. According to functional theory, as long as the kernel function of $K(x, y)$ satisfies Mercer condition, you can achieve linear transformation, the formation of classification function is:

$$
f(x)=\operatorname{sgn}\left\{\left(\sum_{i=1}^{n} a_{i}^{*} y_{i} K\left(x_{i}, x\right)+b^{*}\right)\right\}
$$

Where $a_{i}^{*}$ is the Lagrange multiplier for classification, $b^{*}$ is the kernel function for classification threshold. Commonly used kernel $K\left(x_{i}, x\right)$ functions are polynomial kernel function, radial basis function, Sigmoid kernel function. Select a different kernel functions to get different support vector machine classifier. This choice by comparing polynomial kernel function belongs to the global kernel function, has global feature that allows data points that are far apart can affect the value of the kernel function, can meet the requirements. Polynomial kernel function:

$$
K\left(x_{i}, x\right)=\left[\left(x_{i} \cdot x\right)+1\right]^{q}
$$

Wherein $x_{i}$ is the input feature vector, $\mathrm{q}$ is the order. Support vector machine classification algorithm is divided into: "binary" Support Vector Machine (BT-SVM), it Can be seen that the traditional method of binary tree method avoids the situation can not be separated, and only construct k-1 个 SVM classifier, the test does not necessarily need to calculate all of the classifier discriminant function, which can save test time, while improving the training and test speed.

\section{The Amount of Energy Law}

Testing according to the signal $x(t)$ and known theoretical knowledge, without considering the actual dimensions of the case $x^{2}(t)$, the vibration signal is the time integral of the square of the known signal energy,

$$
Q(i)=\int_{-\infty}^{+\infty}|x(t)|^{2} d t
$$

When the signal $x(t)$ is discrete signals, the signal energy interval signal value added and,

$$
Q(i)=\sum_{t=0}^{n}\left|x^{2}(t)\right| \Delta t
$$

Where $\Delta t$ is the sampling interval, sampling interval in this article $0.1 \mathrm{~ms}$, for the entire range of the total energy of the signal on the segment is $Q(i)$.

For example, the fault signal by EMD decomposition obtained after a series of key information $c_{1}(t), c_{2}(t) \ldots c_{n}(t)$ that contains the failed IMF components, the IMF component from high frequency 
components contained in the end, the circuit breaker failure occurs division closing different mechanical vibration signal generated by the energy value of IMF there are obvious differences.

\section{Empirical Mode Decomposition Energy of Mo Extraction And Classification Process}

The signal will be collected first EMD decomposition, the IMF components and extracts the feature vector fault signal input learn good in SVM classification.

(1) The raw vibration signal with EMD, take the main fault information including the first $n$ IMF components, the paper take the first six IMF components.

(2)Calculated for each IMF (discrete signal) component of total energy $Q(i)$ :

$$
Q(i)=\sum_{t=0}^{n} c_{i}^{2}(t) d t \quad(n=5000)
$$

(3)The IMF component of the total energy of the normalization process:

$$
E_{\mathrm{i}}=\frac{Q(i)}{E}
$$

Where the total energy of the IMF components, the total energy of the original vibration signal.

(4) IMF component to the total energy of structural elements feature vector $\mathrm{T}$ :

$$
T=\left[E_{1}, E_{2}, \ldots, E_{n}\right]
$$

(5)Using the "binary" Support vector machine as classifier, using training samples of fault feature vector training SVM, determine the parameters of SVM classifier.

(6)The failure of the test sample input trained SVM feature vectors in order to determine the output of SVM classifier breaker vibration signal failure type.

\section{Analysis of Experimental Results}

\section{1breaker Vibration Signal EMD Decomposition and Feature Extraction}

In this paper, KL HVB type $12 \mathrm{KV}$ vacuum circuit breaker to do the test, using the Shanghai casting Swiss company YD-1 type acceleration sensor, acceleration reaction force change, range $0-1000 \mathrm{~m} / \mathrm{s} 2$, sensor installed in the circuit breaker operating mechanism outside the vertical direction. Extraction vacuum circuit breaker in case of no-load condition and two normal fault condition vibration signal. Namely: normal (a1, a2), closing solenoid jam (b1, b2) and the spindle jam fault (c1, c2) signal,This can expect the three vibration signal waveform has a different energy transmission capacity, whereby when the signal is decomposed into different time scales, different characteristics can be obtained.

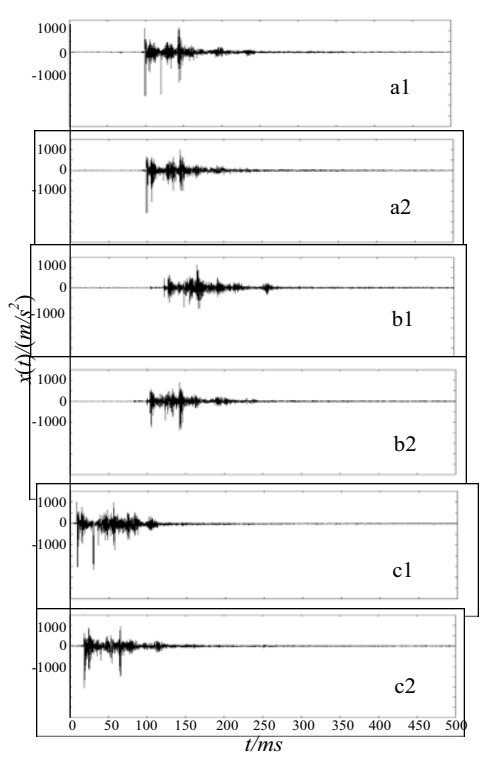

Fig. 2 Normal and Fault signals

Using formula (11) were calculated in the normal state, the switch magnet and the spindle jam fault energy value decomposition of IMF, as the main energy vibration signal and the amount of information generated in the IMF components are the first few components of through experiments and data processing can be drawn, the signal energy is concentrated on the first six IMF components, viz. Here, as shown in Table 1, wherein, E1-E6 is calculated from the information contained signal IMF1-IMF6 principal eigenvectors as a test of SVM input signal, the process is the tracking signal generated by the energy distribution a process.

\subsection{SVM Fault Status Classification}

In this paper, "binary" Support vector machine classification algorithm, set to a normal state, the closing magnet jam fault is 2 , the spindle jam fault is 3 . By analyzing the feature vectors, taking into account the experience. This classification kernel function using polynomial kernel, the order q Take 2 , penalty coefficient $\mathrm{C}$ take 100 to 10 sets of data for each sample the amount of energy range calculation.

Classification process conducted by Equation 5 to 10 have done each sample set of training data, for example, constitute a three sample classification. The first category is the second positive type, the first and third types is negative class training to obtain SVM1. Then the first category as being Class III negative class training to get SVM2. Kernel function of the inner product of a matrix composed of 30 groups of data. The classification process is counted twice Lagrange multipliers and are shown in Table 1, to obtain two kinds of support vector machines SVM1 and SVM2, thus SVM training is complete 
Tab.1 Experiment results

\begin{tabular}{|c|c|c|c|c|c|c|c|}
\hline & $E_{1}$ & $E_{2}$ & $E_{3}$ & $E_{4}$ & $E_{5}$ & $E_{6}$ & $\begin{array}{c}\text { Classification } \\
\text { results }\end{array}$ \\
\hline $\begin{array}{c}\text { Normal } \\
\text { signal I (1) }\end{array}$ & 0.4039 & $\begin{array}{l}0.322 \\
8\end{array}$ & 0.1244 & $\begin{array}{c}0.082 \\
0\end{array}$ & $\begin{array}{c}0.024 \\
2\end{array}$ & $\begin{array}{l}0.02 \\
27\end{array}$ & 1 \\
\hline $\begin{array}{c}\text { Normal } \\
\text { signal I (2) }\end{array}$ & 0.4498 & $\begin{array}{l}0.265 \\
7\end{array}$ & 0.1344 & $\begin{array}{l}0.088 \\
7\end{array}$ & $\begin{array}{l}0.025 \\
5\end{array}$ & $\begin{array}{l}0.02 \\
40\end{array}$ & 1 \\
\hline $\begin{array}{c}\text { Normal } \\
\text { signal I (3) }\end{array}$ & 0.4283 & $\begin{array}{c}0.303 \\
4\end{array}$ & 0.1195 & $\begin{array}{l}0.090 \\
5\end{array}$ & $\begin{array}{l}0.022 \\
8\end{array}$ & $\begin{array}{l}0.02 \\
23\end{array}$ & 1 \\
\hline $\begin{array}{l}\text { Fault signal } \\
\text { II (1) }\end{array}$ & 0.6057 & $\begin{array}{l}0.244 \\
6\end{array}$ & 0.0715 & $\begin{array}{l}0.027 \\
8\end{array}$ & $\begin{array}{l}0.020 \\
2\end{array}$ & $\begin{array}{l}0.01 \\
63\end{array}$ & 2 \\
\hline $\begin{array}{c}\text { Fault signal } \\
\text { II (2) }\end{array}$ & 0.6467 & $\begin{array}{l}0.217 \\
4\end{array}$ & 0.0918 & $\begin{array}{l}0.016 \\
5\end{array}$ & $\begin{array}{l}0.012 \\
9\end{array}$ & $\begin{array}{l}0.00 \\
58\end{array}$ & 2 \\
\hline $\begin{array}{c}\text { Fault signal } \\
\text { II (3) }\end{array}$ & 0.6317 & $\begin{array}{c}0.233 \\
1\end{array}$ & 0.0834 & $\begin{array}{l}0.018 \\
0\end{array}$ & $\begin{array}{c}0.015 \\
6\end{array}$ & $\begin{array}{l}0.01 \\
15\end{array}$ & 2 \\
\hline $\begin{array}{l}\text { Fault signal } \\
\text { III(1) }\end{array}$ & 0.5658 & $\begin{array}{l}0.246 \\
3\end{array}$ & 0.0867 & $\begin{array}{l}0.054 \\
3\end{array}$ & $\begin{array}{l}0.021 \\
0\end{array}$ & $\begin{array}{l}0.01 \\
65\end{array}$ & 3 \\
\hline $\begin{array}{l}\text { Fault signal } \\
\text { III(2) }\end{array}$ & 0.5384 & $\begin{array}{l}0.271 \\
5\end{array}$ & 0.0843 & $\begin{array}{l}0.050 \\
9\end{array}$ & $\begin{array}{c}0.025 \\
3\end{array}$ & $\begin{array}{l}0.01 \\
95\end{array}$ & 3 \\
\hline $\begin{array}{l}\text { Fault signal } \\
\text { III(3) }\end{array}$ & 0.5500 & $\begin{array}{l}0.255 \\
8\end{array}$ & 0.0857 & $\begin{array}{c}0.053 \\
1\end{array}$ & $\begin{array}{l}0.023 \\
9\end{array}$ & $\begin{array}{l}0.01 \\
73\end{array}$ & 3 \\
\hline
\end{tabular}

\section{Conclusion}

In this paper, empirical mode decomposition method breaker vibration signal decomposition, the total energy use of IMF as a feature vector representation breaker failure, and as the input vector of SVM, the use of "one to one multi-category" Support Vector Machine classification mechanism for training, classification results obtained. The experimental results show that this method can effectively extract the circuit breaker fault vibration signal feature vector and can correct for state recognition and fault classification.

\section{References}

[1] Wu Jianwen,Lian Shijun. A kind of fault isolation and lockout features microprocessor-based protection boundary switch [C].2007 Anual Conference on Relay Protection and Automation in China.2007.

[2]Sun Yihang, $\mathrm{Wu}$ Jianwen.Design on Intelligent Integrated Controller of Circuit Breaker Based on Dual-core CPU.2011 1st International Conference on Electric Power Equipment-Switching Technology[C],2011,315-318

[3] Wu Jianwen,Sun Yihang Zhang Luming,et al. A range of failure is isolated circuit breaker controller [P].201110104084.X.

[4]Huang $\mathrm{N}$ E,et a1..The empirical mode decomposition and the Hilbert spectrum for nonlinearand non-stationary time serie analysis[J] Proc.R.Soc.Lond.A 1998 45(4):903-995.

[5] Li Tianyun, Zhao Yan, Ji Xiaohui. Application of hht method for analysis of fault signal in electric power system [J]. Transactions of China Electrotechnical Society, 2005, 20(6): 87-91(in Chinese).

[6] Yang Y, Yu D J, Cheng J S. A roller bearing fault diagnosis methodbased on EMD energy entropy and ANN [J]. Journal of Sound andVibration, 2006(294): 269-277.

[7] Cheng J S, Yu D J, Tang J S, et al. Application of frequency family separation method based upon EMD and local Hilbert energy spectrum method to gear fault diagnosis $[\mathrm{J}]$. Mechanism and Machine Theory, 2008(43): 712-723.

[8]Loh $\mathrm{CH}$, et a1.Application of the empirical mode decomposition-Hilbert spectrum method to identify near-fault ground-motioncharacteristics and structural nssdonses[J].Bulletin of theseism gical society of america 2001(91):1339-1357

[9] Cheng Junsheng.. Studying and Application of Hilbert-Huang Transform in the Fault Diagnosis of Rotating Machinery [D],Hu nan Univ ersity.,2005. 